\title{
ZWISCHEN TANNENBERG UND THORN: KRISENBEWÄLTIGUNG DES RATES DER ALTSTADT ELBING I. J. 1410
}

\section{田} oruff nome mir berath mit der gemeyne unde worden des noch unserm besten dirkennen gantz eyns, das wir deme koninge van Polen eyme kristen unde nicht Wittauten eyme busen kristen huldigen wulden uff eyne vryst, als do leyder die luffe czusageten. ${ }^{1}$ Mit diesen Worten entschuldigte der Stadtrat vor dem Hochmeister-Statthalter Heinrich von Plauen die Unterwerfung der Altstadt Elbing unter die Herrschaft des polnischen Königs nach der Schlacht bei Tannenberg vom 15. Juli 1410. Das entsprechende Schreiben hat der Rat nach dem Abbruch der polnischen Belagerung der Marienburg vom 19. September 1410 am 9. Oktober 1410 an Plauen abgesandt.

Der Forschung ist es seit langem bekannt, dass die Rückkehr Elbings unter die Ordensherrschaft im Herbst 1410 ohne Vorbedingung stattfand. Im Gegensatz dazu standen Thorn und Danzig, die sich in dem noch andauernden Krieg zwischen dem Deutschen Orden und Polen und Litauen Neutralität ausbedungen hatten. ${ }^{2}$ Es steht sicherlich außer Frage, dass die Anwesenheit des vor Elbing lagernden Ordensheeres den Entschluss des Rates früher reifen ließ. Die vorbehaltlose Unterstützung der Sache des Ordens aber muss noch andere Ursachen haben. Diesen gilt es im Folgenden nachzuspüren. Es soll vornehmlich anhand des noch unveröffentlichten Nachrichtenmaterials zu der städtischen Führungsschicht aus der Zeit kurz vor der Schlacht bei Tannenberg bis zum Thorner Friedensschluss von 1411 geschehen. Der Ablauf der Ereignisse, den Markian Pelech in seinem Aufsatz von 1987 über die Teilnahme der Altstadt Elbing am Großen Krieg zu-

1 Gedruckt im Anhang von M. Pelech, Die Teilnahme der Altstadt Elbing am Grossen Krieg (1409-1411), Beiträge zur Geschichte Westpreußens 10 (1987), S. 49-65, hier 65.

2 E. Carstenn, Geschichte der Hansestadt Elbing, Elbing 1937, S. 188; O. Stavenhagen, Livland und die Schlacht bei Tannenberg, Baltische Monatsschrift 44 (1902), S. 235-381, hier 368. 
sammengefügt hat, stellt dabei den Orientierungsrahmen. Im ersten Schritt soll der Versuch stehen, das Führungspersonal von um 1380 bis zur TannenbergSchlacht vor allem im Hinblick auf seine Nähe oder Ferne zur Landesherrschaft vorzustellen. Besonders vor dem Hintergrund von politisch motivierten Mordanschläge auf zwei Elbinger Bürgermeister gilt es danach im zweiten Schritt, in ähnlicher Weise das Führungspersonal nach der Schlacht bis etwa zum Frieden von Thorn von 1411 zu skizzieren. Im dritten Schritt soll die Bewältigung der politischen Krise nachgezeichnet werden. Am Schluss steht eine kurze Zusammenfassung der wesentlichen Ergebnisse.

\section{Das Elbinger Führungspersonal von um 1380 bis zur SCHLacht beI}

TANNENBERG VON 1410

Elbing gehörte zu den wenigen preußischen Städten, in denen lübisches Recht galt, wenn auch mit Einschränkungen. Demnach setzte sich der Stadtrat im Idealfall aus 22 Ratmannen und vier Bürgermeistern zusammen. Rund zwei Drittel der lebenslänglich kooptierten Ratsmitglieder bildeten den regierenden oder sitzenden Rat, der Rest den sog. alten Rat. Beide Ratsteile traten unter der Bezeichnung "gemeiner Rat" zusammen. Der Austausch zwischen sitzendem und altem Rat erfolgte gewöhnlich nach Ablauf des dritten Jahres. ${ }^{3}$

Spätestens im Laufe des 14. Jahrhunderts hatte sich die Gewohnheit verfestigt, dass sich der Rat insbesondere aus dem Kreise bestimmter Berufsgruppen oder Gewerbetreibenden erneuerte. Prosopographische Untersuchungen anhand des Elbinger Kriegsbuches von 1383 bis 1409 legen den Schluss nahe, dass vornehmlich wirtschaftlich erfolgreiche Kaufleute, Schiffer, Brauer und Fischhändler Ansprüche auf die Ratsfähigkeit begründen konnten. Die Mitglieder handwerklicher Berufe galten im Einklang mit dem lübischen Recht dagegen als nicht ratsfähig. ${ }^{4}$ Es versteht sich beinahe von selbst, dass die Zugehörigkeit zu einer bereits ratsfähigen Familie oder die Einheirat in seine solche Familie die Zuwahl in den Rat zu erleichtern pflegte. ${ }^{5}$ Identitätsstiftend auf die ratsfähigen Familien wirkte dabei die besondere Verehrung des heiligen Ritters und Drachentöters Georg. Sie fand ihren Ausdruck in der Georgsbruderschaft, in der Georgsbank im Artus-

3 M. Töppen, Elbinger Antiquitäten. Ein Beitrag zur Geschichte des städtischen Lebens im Mittelalter, Marienwerder 1870, S. 184; R. Czaja, Urzędnicy miejscy Elblaga do 1524 roku [Städtische Amtsträger in Elbing bis zum Jahr 1524], Elbląg 2010, S. 7-22.

4 Czaja, Urzędnicy (wie Anm. 3), S. 9.

5 Demnächst D. Heckmann, Schiffer der Altstadt Elbing um 1400: Rückgrat für Wirtschaft und Krieg. 
hof, in der Sorge für das St.-Georgs-Hospital vor dem Markttor und in einer freilich erst zu 1478 belegten Stiftung des Georg-Altars in der Stadtpfarrkirche ${ }^{6}$ sowie nicht zuletzt in der Nachahmung der ritterlichen Lebensweise. Eine große Rolle spielte gerade dabei die Rezeption der Artuslegende ${ }^{7}$, die mit dem Artushof und dem von diesem gegründeten Junkergarten ihren sichtbarsten Ausdruck fand.

Im Bürgermeisteramt ${ }^{8}$ der Altstadt Elbing sind zwischen 1380 und 1410 Hartwig d. Ä. Betke, Tidemann Rover, Johann d. J. Stolte, Johannes d. Ä. von Hervord, Gerhard von Thorn, Heinrich Damerow, Johannes d. M. Trugehorn, Arnd d. M. Rover, Johann d. M. von Thorn, Werner Wesseling, Lifhard d. M. von Hervord, Johann d. M. Rode und Johannes Werner' nachweisbar. Zu den herausragenden gemeinsamen Merkmalen dieser Personengruppe gehört, dass die genannten Bürgermeister ausnahmslos Großkaufleute waren und einen Teil ihrer Einkünfte aus dem Besitz von Liegenschaften in Elbing und in der Stadtmark sowie aus Hypotheken- und Rentengeschäften bezogen. Immerhin gestattet es die Überlieferungslage, innerhalb dieser Gruppe Unterschiede heraus zu arbeiten. Demnach pflegten Hartwich d. Ä. Betke, Johan d. J. Stolte, Heinrich Damerow, Johann d. M. von Thorn, Lifhard d. M. von Hervord, Johann d. M. Rode und Johannes Werner ein besonders enges Verhältnis zur Landesherrschaft zu unterhalten. Als Indikatoren hierfür lassen sich Geschäftsbeziehungen mit dem Orden, die Beteiligung an diplomatischen Missionen des Ordens oder gar die Mitgliedschaft im hochmeisterlichen Rat benennen. So stand Johannes Werner ${ }^{10}$ mit dem Königsberger Großschäffer wegen des Tuchhandels und mit dem Komtur von Christburg wegen des Getreidehandels im Geschäftskontakt. Auch Johann d. J. Stolte pflegte die Geschäftspartnerschaft mit dem Christburger Komtur. Außerdem war er Nutznießer von dem Kapitalbedarf französischer oder flandrischer Preußenfahrer. ${ }^{11}$ Der Ma-

6 P. Oliński, Fundacje mieszczańskie w miastach pruskich w okresie średniowiecza i na progu czasów nowożytnych [Bürgerstiftungen in preußischen Städten im Mittelalter und zu Beginn der Neuzeit], Toruń 2008, S. 239 f.

7 Siehe dazu S. Selzer, Artushöfe im Ostseeraum. Ritterlich-höfische Kultur in den Städten des Preußenlandes im 14. und 15. Jahrhundert (Kieler Werkstücke, Reihe D: Beiträge zur europäischen Geschichte des späten Mittelalters 8), Frankfurt/Main u. a. 1996; und E. Pilecka, Średniowieczne dwory Artusa w Prusach. Świadectwo ksztattowania się nowej świadomości mieszczańskiej [Mittelalterliche Artushöfe in Preußen. Zeugnis der Gestaltung eines neuen städtischen Bewusstseins], Toruń 2005.

8 Zusammengestellt nach Czaja, Urzędnicy (wie Anm. 3).

9 Nowa ksiega rachunkowa starego miasta Elblaga, Tl. 1: 1404-1410, Tl. 2: 1411-1414, hrsg. v. M. Pelech (Fontes 72-73), Warszawa-Poznań-Toruń 1987-1989, hier Tl. 1, Nr. 999.

10 Siehe dazu „Werner, Johanes d. Ä., in: Elbinger Kriegsbuch (1383-1409). Rechnungen für städtische Aufgebote (Veröffentlichungen aus den Archiven Preußischer Kulturbesitz 68), bearb. v. D. Heckmann unter Mitbearb. v. K. Kwiatkowski, Köln-Weimar-Wien 2013, S. 415-417.

11 Siehe dazu „Stolte, Johan d. J“, in: ebd., S. $394 \mathrm{f}$. 
rienburger Großschäffer beteiligte sich an den Kosten für die Gesandtschaftsreisen von Heinrich Damerau von $1406 .{ }^{12}$ Lifhard d. M. von Hervord handelte mit dem Ordensdiplomaten Dietrich von Logendorff ${ }^{13}$ und dem Danziger Stadtschreiber Krolau in London den englisch-preußischen Handelsvertrag vom 4. Dez. 1409 aus. ${ }^{14}$ Hochmeister Konrad Zöllner von Rothenstein verlieh am 11. Oktober 1383 Hartwig Betke ob seiner treuen Dienste 11 Hufen und 9 Morgen Land in Hansdorf zu kulmischem Recht. ${ }^{15}$ Und Johann d. M. von Thorn zählte zu den Familiaren des pomesanischen Bischofs Johann Mönch, eines gebürtigen Elbingers, und saß obendrein im Rat des Hochmeisters. ${ }^{16}$

Die Überlieferungslage gestattet es, daneben eine zweite Riege von Bürgermeistern auszumachen. Diese kennzeichnet sich hauptsächlich dadurch, dass ihre Angehörigen ohne besondere Beziehungen zum Orden waren, zumindest ließen sich solche bislang nicht nachweisen. In diese zweite Riege lassen sich Tidemann Rover, Johannes d. Ä. von Hervord ${ }^{17}$, Gerhard von Thorn ${ }^{18}$, Johannes d. M. Trugehorn $^{19}$ und Werner Wesseling ${ }^{20}$ einordnen. Selbst wenn weitere Quellenfunde den Eindruck erhärten sollten, dass diese Leute keine besonderen Beziehungen zum Orden unterhielten, wäre es unzulässig, ihnen von vorne herein eine Gegnerschaft zum Orden zu unterstellen. Allenfalls ließe sich ein gewisser Abstand zur Landesherrschaft beobachten, der aber einer konstruktiven Zusammenarbeit mit dem Orden beispielsweise in Handelsangelegenheiten nicht entgegengestanden haben dürfte. Lediglich Arnd d. M. Rover könnte ein Sonderfall dargestellt haben. Eindeutige Quellenbelege dafür gibt es freilich nicht. Die fehlenden Nachweise einer persönlichen Beteiligung an den Litauenzügen des Ordens oder an den Landwehraufgeboten mögen aber in diese Richtung deuten. Arnds Teilnahme an der Befreiung des Herzogs von Geldern im Frühjahr 1389, der auf seinem Weg nach Preußen im neumärkischen Falkenburg gefangen gehalten wurde ${ }^{21}$, dürfte dem nicht widersprechen. Die Elbinger scheinen die Befreiungsaktion mehr als gesellschaftliches Großereignis denn als Kriegszug empfunden zu haben. Galt es doch, den eigenen Reichtum und das damit verbundene Ansehen öffentlich zur

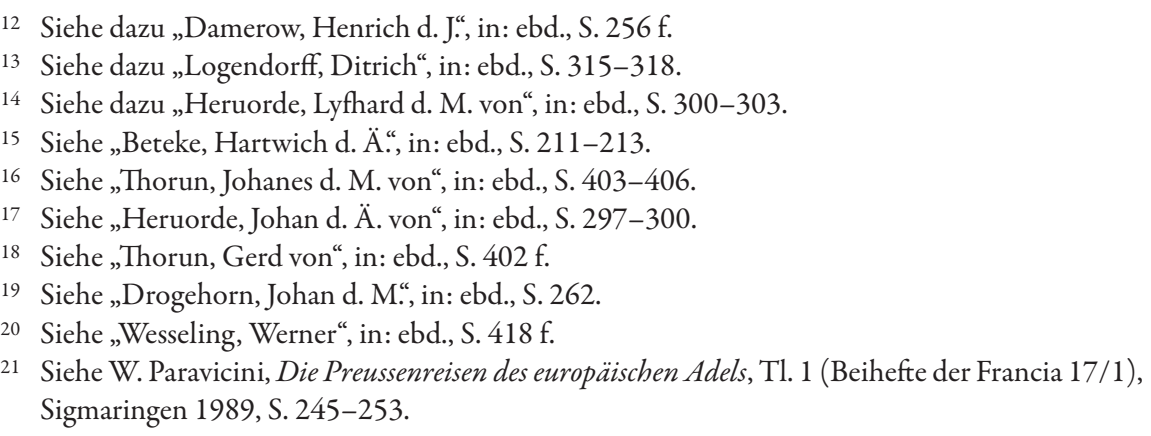

21 Siehe W. Paravicini, Die Preussenreisen des europäischen Adels, Tl. 1 (Beihefte der Francia 17/1), Sigmaringen 1989, S. 245-253. 
Schau zu stellen. Dementsprechend trat Arnd mit drei Streithengsten und drei Wäppnern auf. Andere aus der städtischen Oberschicht standen ihm in Nichts nach. Es gab sogar Leute wie Henrich Mekelborg und Claus Cornegil, die damals als Freiwillige mitgezogen sind.

Den bisherigen Beobachtungen nach verfestigt sich der Eindruck, dass von den 13 Personen der Altstadt Elbing, die zwischen 1380 und 1410 im Bürgermeisteramt nachweisbar sind, alleine sieben als Vertraute des Ordens gelten können. Bei vieren lässt sich ein gewisser Abstand zur Landesherrschaft unterstellen und lediglich bei einem Bürgermeister, nämlich bei Arnd d. M. Rover, vermag ein schwacher Hinweis seine ordenskritische Haltung anzudeuten.

Noch spärlicher als bei den Bürgermeistern sprudeln die Quellen bei den rund 50 Ratmannen, die zwischen 1380 und 1409 nachweisbar sind. Ungeachtet dessen überwiegt auch hier der Eindruck, als seien die einfachen Ratmannen loyale Ordensuntertanen gewesen, zumal die Quellen keinen gegenteiligen Anhaltspunkt liefern. Als besonders ordensnah können Claus d. J. Wulff, Johann d. J. Raw, Johann und Heinrich Krutzeburg oder Claus d. J. Dirgarde gelten.

\section{DAs Elbinger FÜHRUngspersonal IN DEN JAHREN 1410 UND 1411}

Für das Jahr 1410 hat Czaja 27 Ratmannen ermittelt, darunter die Bürgermeister Johann von Thorn, Lifhard von Hervord, Johann Rode und Arnd Rover. Von diesen 27 Ratsgliedern sind mit Sicherheit Heinrich Altmann, Johann d. J. von Hervord, Tidemann von der Wyde und Johann Raw in der Schlacht bei Tannenberg vom 15. Juli 1410 geblieben. Bei Bertram Betke und Johann Krutzeburg ist es zumindest hoch wahrscheinlich, dass auch sie zu den Gefallenen zu rechnen sind. Was aber den Tod der beiden Bürgermeister Johann d. M. von Thorn und Johann Rode angeht, so gehen die Meinungen in der Forschung auseinander. Während Max Töppen noch davon ausging, die beiden seien auf dem Schlachtfeld bei Tannenberg umgekommen ${ }^{22}$, vermutete Edward Carstenn, Hochmeister Heinrich von Plauen habe sie beseitigen lassen. ${ }^{23}$ Und Marian Biskup hält sie gar für Opfer eines Raubmordes. ${ }^{24}$ Vorsichtiger hat sich Semrau dazu geäußert, indem er

22 A. Semrau, Johann von Thorun, Bürgermeister der Altstadt Elbing, † 1410, Mitteilungen des Coppernicus-Vereins für Wissenschaft und Kunst zu Thorn 31 (1923), S. 37-42, hier 41.

23 Carstenn (wie Anm. 2), S. 470 Anm. 6.

24 M. Biskup, Zwei Elbinger Kaufleute und Ratsherren (Mitte des 14. Bis Anfang des 15. Jahrhunderts): Johann von Volmenstein und Johann von Thorn, in: Akteure und Gegner der Hanse - Zur Prosopographie der Hansezeit (Hansische Studien IX), hrsg. v. D. Kattinger, H. Wernicke unter Mitwirkung v. R.-G. Werlich, Weimar 1998, S. 93-107, hier 107. 
schrieb: Näheres lässt sich wenigstens zur Zeit nicht ermitteln. ${ }^{25}$ Pelech meinte sogar, dass Johann von Thorns Schicksal ungeklärt bleiben muss. ${ }^{26}$ Aus der Zusammenschau der wenigen vorhandenen Nachrichten drängt sich jedoch folgendes Bild auf: Johann von Thorn und Johann Rode mussten zwischen dem Abzug des polnisch-litauischen Belagerungsheeres vor der Marienburg vom 19. September 1410 und der Kür Heinrichs von Plauen zum Hochmeister, die am 9. November desselben Jahres stattgefunden hatte, zu Tode gekommen sein. Einer Eintragung aus dem Elbinger Kämmereibuch nach führten nämlich Johann von Thorn und Claus Wulff die Elbinger Gesandtschaft an, die Plauen noch in seiner Eigenschaft als Komtur von Schwetz in der Marienburg Ehrengeschenke überreicht hatte. ${ }^{27}$ Hinzu kommt die Aufzeichnung einer Elbinger Geldausgabe zu Gunsten der Witwe von Johann Rode aus dem Jahr 1413. Hiernach habe Johann Rode auf einer Fahrt nach Neuenburg van den Polan Schaden genommen. ${ }^{28}$ Die Nachricht, dass die Altstadt Elbing ein gemeinsames Requiem für die Gefallenen der Tannenberg-Schlacht und der beiden getöteten Bürgermeister begehen lie $\aleph^{29}$, dürfte mittelbar belegen, dass nicht nur Johann Rode, sondern auch Johann von Thorn von den Polen Schaden genommen hatte.

Dass Johann von Thorn und Johann Rode sich nur schwer mit dem Verlust der Ordensherrschaft abfinden konnten, entging dem polnischen König nicht. Wladislaw Jagiello musste Elbing sogar ernstlich drohen, damit die Stadt sich zu Huldigungsverhandlungen bereitfand. Die Verhandlungen führten Johann von Thorn und Johann Werner ${ }^{30}$, der übrigens seit $1414 \mathrm{zu}$ den geschworenen Räten des Hochmeisters zählte ${ }^{31}$. Auf dem Städtetag vor Marienburg vom 10. August, den auch Thorn, Braunsberg und Danzig besendet hatten, handelten Johann von Thorn und Johann Rode zusätzliche Privilegien für die Altstadt Elbing aus. ${ }^{32}$ Kurz darauf fand sich Johann Rode wiederum beim König ein, um anschließend zu den Vertretern anderer preußischer Städte, die sich in Neuenburg versammelt hatten, zu stoßen. Dort sei her Iohan zere beschidiget worden, wie es in der Quelle heißt. ${ }^{33}$ Polnischerseits gab es demnach genügend Beweggründe, solche politisch missliebi-

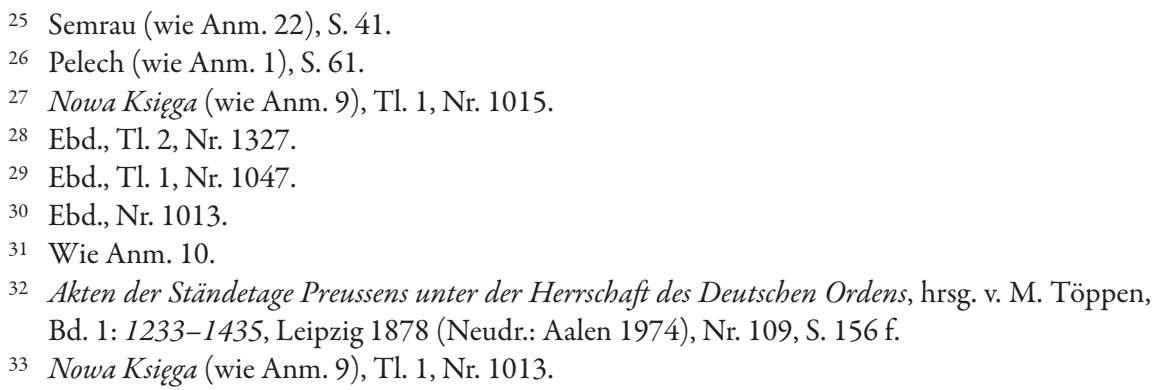


gen Leute - vielleicht unter dem Deckmantel des Straßenraubes - beseitigen zu lassen.

Die politische Führung in Elbing hatte im Krisenjahr 1410 auch den Rücktritt von Lifhard von Hervord vom Bürgermeisteramt zu verkraften. Wie bereits erwähnt, gehörte Lifhard mit dem Ordensdiplomaten Dietrich von Logendorff und dem Danziger Stadtschreiber Johann Krolau zu den preußischen Unterhändlern, die am 4. Dezember 1409 mit den Beauftragten des englischen Königs in London den preußisch-englischen Handelsvertrag ausgehandelt hatten. ${ }^{34}$ Von daher ist die Vermutung naheliegend, dass es die Unzufriedenheit mit den wesentlichen Vertragsbedingungen war, die Lifhard wohl noch im Frühjahr 1410 veranlasst hatte, sein Bürgermeisteramt nieder zu legen. ${ }^{35}$ Seinen Platz hat wohl Johann Werner eingenommen. ${ }^{36}$

Der Elbinger Rat scheint aber erst 1411 damit begonnen zu haben, die kriegsbedingten Lücken in seinen Reihen mit neuen Mitgliedern zu schließen. Für die zweite Hälfte des Jahres 1410 lassen sich indes Umschichtungen und Kumulierungen von Ratsaufgaben erkennen. So hatte der neu in den Rat kooptierte Claus von Krossen den Befehl über das Landwehraufgebot inne, das vor der Schlacht von Tannenberg zur Deckung von Thorn eingesetzt war. Und nach der Schlacht war er mit den Aufgaben des Kriegsschäffers betraut, die dem gefallenen Heinrich Altmann oblegen waren. Aus persönlichen Gründen hatte Claus von Krossen während der Winterreise von 1400 nach Samaiten, die Aufgebotsverpflichtung von Heinrich Altmann übernommen, sodass diese Annahme nicht unbegründet erscheint. Innerhalb des Rates genoss Claus ein derartiges Vertrauen, dass er Ende des Jahres $1410 \mathrm{zu}$ den Vorverhandlungen für den 1. Thorner Frieden vom 1. Februar 1411 entsandt wurde. ${ }^{37}$ Umschichtungen von Ratsaufgaben sind vor allem hinsichtlich des Befestigungsbaues zu erkennen. Der Rat beauftragte gleich zwei seiner Mitglieder mit der Schafferei für die Bollwerke, nämlich den Ältermann der mit dem Bürgerschießgarten eng verbundenen Blasiusbruderschaft Claus Kustrate und den Kaufmann Claus d. J. Volmersten. Auch diese beiden Schäffer gehörten erst seit 1410 dem Rat an. Nachdem just 1409 und 1410 beträchtliche Teile der

34 E. Weise, Die Staatsverträge des Deutschen Ordens in Preußen im 15. Jahrbundert, Bd. 1, Marburg 2 1970, Nr. 60; Nowa Ksiegga (wie Anm. 9), Tl. 1, Nrn. 766, 769 f., 783, 1002; Die Recesse und andere Akten der Hansetage 1256-1430, Bd. 5, bearb. v. K. Koppmann, Leipzig 1880 (Neudr.: Hildesheim-New York 1975), Nr. $655 \$ 20$; S. Jenks, England, die Hanse und Preußen. Handel und Diplomatie, 1377-1474, Bd. 1-3 (Quellen und Darstellungen zur Hansischen Geschichte, N.F. 38,1-3), Köln-Wien 1992, S. 539-549.

35 Nowa Ksiega (wie Anm. 9), Tl. 1, Nrn. 741, 743 f., 746.

36 Czaja, Urzędnicy (wie Anm. 3), S. 89, Anm. 385.

37 Siehe „Krossen, Claus von“ (wie Anm. 10), S. 244-246. 
städtischen Ummauerung eingestürzt waren ${ }^{38}$, duldete die Erledigung ihrer Aufgaben keinen Aufschub.

Mit der gewohnten Kür des Rates an Petri Stuhlfeier 1411 (22. Februar) traten Niclos Busdorff ${ }^{39}$, Tidemann von Monster, Claus Birsmit ${ }^{40}$ und Heinrich Palborn auf den Plan. Unter den neuen Ratsgliedern heben sich Tidemann von Monster und Heinrich Palborn durch ihre Nähe zum Orden ab. Mit Sicherheit darf Tidemann als enger Vertrauter des Ordens gelten. Er war nämlich zwischen 1398 und 1402 Schreiber des Königsberger Großschäffers und danach bis 1410 Elbinger Wirt des Komturs von Brandenburg gewesen. Mit Hermann Huxer, dem Thorner Lieger des Großschäffers, unterhielt Tidemann zweitweise eine Handelsgesellschaft. Zu Tidemanns Kunden zählte übrigens auch der Komtur von Balga. ${ }^{41}$ Auch bei Heinrich Palborn gibt es deutliche Hinweise auf enge Beziehungen zur Ordensleitung. ${ }^{42}$ Insoweit wäre es nicht verfehlt, in den beiden einen gewissen Ersatz für die 1410 ermordeten Johann von Thorn und Johann Rode zu sehen. Ungeachtet dessen hatte allem Anschein nach Hochmeister Heinrich von Plauen im Jahre 1413 die Aufnahme des Englandfahrers und Bierhändlers Claus Bruggemann in den Rat durchgesetzt, was der Rat nach Plauens Sturz allerdings wieder rückgängig machen ließ ${ }^{43}$ Mit Sicherheit sah er in dem Vorstoß Plauens einen unerträglichen Eingriff in die städtischen Freiheiten.

\section{BEWÄLTIGUNG DER POLITISCHEN KRISE}

Da die Skizzen der soeben vorgestellten Mitglieder der Elbinger Führungsschicht nicht einmal für die Zeit nach der verlorenen Schlacht bei Tannenberg nennenswerte Ansätze für eine ordensfeindliche Haltung bieten, gilt es, an dieser Stelle die Ratspolitik in den Ablauf der Ereignisse zwischen dem 15. Juli 1410, dem Datum der Schlacht bei Tannenberg, und dem Abschluss des 1. Thorner Friedens vom 1. Februar 1411 einzublenden. Als Eckdaten für die politische Krise der Altstadt Elbing lassen sich der 22. Juli, der mutmaßliche Tag der Huldigung vor dem polnischen König zu Samrodt bei Mohrungen, und der 9. Oktober 1410 festlegen, auf den die Stadt das eingangs zitierte Entschuldigungsschreiben an Heinrich von Plauen datiert hatte. Dazwischen lag der Städtetag vor Marienburg vom

\footnotetext{
38 Töppen (wie Anm. 3), S. 92; Pelech (wie Anm. 1), S. 52.

39 Siehe „Busdorff, Niclos“ (wie Anm. 10), S. 228 f.

40 Siehe „Birsmit“ (wie Anm. 10), S. 217 f.

41 Siehe „Monstir, Tyde von“ (wie Anm. 10), S. 338 f.

42 Siehe „Palborn, Heinrich“ (wie Anm. 10), S. 348 f.

43 Siehe „Bruggeman, Claus“ (wie Anm. 10), S. 226.
} 
10. August, auf dem Thorn, Elbing, Braunsberg und Danzig dem polnischen König die Erweiterung ihrer Privilegien abgehandelt hatten. Die neu gewonnenen Rechte und Freiheiten scheint die Elbinger Führungsschicht dennoch nicht von ihren Vorbehalten gegenüber der polnischen Herrschaft abgehalten zu haben. Johann Rode hatte diese sicherlich nochmals während der Tagfahrt zu Neuenburg, die Krzysztof Kwiatkowski zu Recht auf die Zeit zwischen dem 20. und 30. August datiert hat ${ }^{44}$, trotz oder gerade wegen einer vorausgegangenen Unterredung mit Wladislaus Jagiello bekräftigt. Rode ist - wie geschildert - damit auf heftigen Widerspruch gestoßen. Elbing hielt an dem eingeschlagenen Kurs fest. Obwohl die Stadt nicht zuletzt aus Furcht vor Plünderungen half, das polnische Belagerungsheer vor der Marienburg mit kriegswichtigen Gütern und Proviant zu versorgen und zudem hochgestellte Persönlichkeiten mit Geschenken ehrte ${ }^{45}$, trat der Rat heimlich in Verbindung zu dem Hochmeisterstatthalter Heinrich von Plauen. Mittelsmänner bei dieser hochverräterischen Handlung waren der Elbinger Schiffer Heinrich Husmann und der Söldnerführer Heinrich d. Ä. von Plauen, der damals mit seinen Reisigen zu Balga lag. ${ }^{46}$ Die Rückkehr Elbings unter die Ordensherrschaft Ende September leitete das Verhalten des litauischen Großfürsten Witold ein: Anfang September entsandte der Rat Johann von Thorn und den Kriegsschäffer Claus von Krossen zum König vor die Marienburg und anschließend zum Großfürsten, der sein Heerlager bei Preußisch Holland aufgeschlagen hatte. ${ }^{47}$ Die Anwesenheit des Kriegsschäffers sowie ein wohl zum 10. September in Frauenburg anberaumter Städtetag ${ }^{48}$ deuten an, dass die Bedrohung durch den Orden insbesondere nach dem Eintreffen des Entsatzheeres unter dem livländischen Marschall Hermann Vincke angeschwollen war. Witold eilte ihm bis an die Passarge entgegen. Von dort zog er sich aber nach Warnungen des ermländischen Bischofs ${ }^{49}$ und wohl auch eines Elbinger Boten, den polnische Bewaffnete bis zur Passarge geleiteten ${ }^{50}$, zum Hauptheer vor der Marienburg zurück, nicht bevor er mit dem Komtur von Goldingen am 8. September einen auf 14 Tage befristeten Waffenstillstand ausgehandelt hatte. Diesen und die gegebene Geleitzusage woll-

44 K. Kwiatkowski, Wyprawa letnia 1410 roku [Der Sommerfeldzug von 1410], in: S. Jóźwiak, K. Kwiatkowski, A. Szweda, S. Szybkowski, Wojna Polski i Litwy z zakonem krzyżackim $w$ latach 1409-1411 [Der Krieg Polens und Litauens gegen den Deutschen Orden 1409-1411], Malbork 2010, S. 238-563, hier 547.

45 Pelech (wie Anm. 1), S. 59.

46 Siehe „Husman, Heinrich“ (wie Anm. 10), S. 308.

47 Kwiatkowski, Wyprawa (wie Anm. 44), S. 546; Nowa Ksiega (wie Anm. 9), Tl. 1, Nr. 1014.

48 Kwiatkowski, Wyprawa (wie Anm. 44), S. 550 f.

49 Scriptores rerum Prussicarum, Bd. 3, hrsg. v. Th. Hirsch, M. Töppen, E. Strehlke, Leipzig 1866 (Neudr.: Frankfurt/Main 1965), S. 79-388, hier 321.

50 Nowa Ksiega (wie Anm. 9), Tl. 1, Nr. 1014. 
ten die livländischen Heerführer nutzen, um mit ihren belagerten preußischen Ordensbrüdern am 14. September in der Marienburg zusammenzutreffen. ${ }^{51}$ Witold traf am 11. oder 12. September vor der Marienburg ein..$^{52}$ Der danach eingeleitete Abzug der litauischen und polnischen Streitkräfte und die damit verbundenen Aufhebung der Belagerung am 19. September hatte keineswegs die sofortige Übergabe der Elbinger Burg durch die polnische und litauische Besatzung zur Folge. Die Bitte des Elbinger Komturs Werner von Tettingen an Heinrich von Plauen vom 29. September um Verstärkung zeigt an, dass er mit der Belagerung der Burg erst wenige Tage vorher ernsthaft begonnen hatte. Die Besatzung harrte immerhin noch rund drei Wochen aus, denn am 25. Oktober gerieten die gegen freien Abzug heimwärts ziehenden Polen mit den Rastenburgern in ein Gefecht. ${ }^{53}$ In dieser ereignisreichen Zeit blieb der Elbinger Rat nicht untätig. Zunächst versuchte er, Plauen und Tettingen gnädig zu stimmen, indem er wohl zwischen dem 19. und 29. September die beiden mit einer Last Bier, die er nach Marienburg schiffen ließ, ehrte. ${ }^{54}$ Bald darauf begann die Stadt, den Orden tatkräftig zu unterstützen. Bei der Belagerung der Elbinger Burg setzte sie beispielsweise eigene Söldner ein. Der Fischhof und die Badstuben gingen damals in Flammen auf. ${ }^{55}$ Dem eingangs zitierten Entschuldigungsschreiben vom 9. Oktober verhalf die Stadt Nachdruck mit einem Aufgebot, das sie zum livländischen Landmarschall ins Kulmerland schickte. ${ }^{56}$ Dort organisierte der Marschall die Belagerung der von polnischen Streitkräften gehaltenen Burg Rehden. ${ }^{57}$ Ein weiteres Aufgebot entsandte der Rat nach Neidenburg zur Verstärkung der dortigen Landwehr. ${ }^{58}$ Ende des Jahres erscheint das Vertrauen zwischen der Ordensleitung und dem Elbinger Rat vollends wiederhergestellt, denn der Elbinger Ratmann Claus von Krossen nahm an den Vorverhandlungen zum Thorner Frieden teil. ${ }^{59}$ Im Kontrast zur Altstadt stand die Politik der Neustadt. Auch wenn hier die Quellenlage nur sehr dürftig ist, lässt sich dennoch erkennen, dass der Rat der Neustadt Elbing sich rasch mit dem neuen Landesherrn ins Benehmen gesetzt hatte; denn noch im Juli 1410 hat-

\footnotetext{
51 Stavenhagen (wie Anm. 2), S. $334 \mathrm{f}$.

52 Kwiatkowski, Wyprawa (wie Anm. 44), S. 548.

53 Pelech (wie Anm. 1), S. 58 f.; und GStA PK, XX. HA Hist. StA Königsberg, OBA Nr. 1340 (zu 1410 Sept. 29).

54 Pelech (wie Anm. 1), S. 59, und Nowa Księga (wie Anm. 9), Tl. 1, Nr. $1005 .$.

55 Nowa Ksiega (wie Anm. 9), Tl. 1, Nr. 1064.

56 Ebd., Nr. 1136.

57 K. Kwiatkowski, Neue Quellen aus dem Kreis des Deutschen Ordens zum Krieg von 1410-1411, Zapiski Historyczne 75 (2010), 4, S. 601-646, hier 627 f., Nr. 17 (zu 1410 Okt. 15).

58 Nowa Ksiega (wie Anm. 9), Tl. 1, Nr. 1135.

59 Ebd., Nr. 1137; s. dagegen Pelech (wie Anm. 1), S. 60, der von einem militärischen Kontingent spricht.
} 
te der polnische König die Neustadt mit den Dörfern Grunau und Neuendorf begabt. ${ }^{60}$ Bald nach der Wiederherstellung der Ordensherrschaft ließ der Elbinger Komtur den Bürgermeister der Neustadt inhaftieren, angeblich wegen der Klage zweier Bürger. So stellte es wenigstens der Hauskomtur in einem Schreiben an den Hochmeister vom 15. Dez. 1410 dar. ${ }^{61}$ Das Lob des polnischen Chronisten Jan Długosz, Elbing habe sich bald nach der Niederlage von Tannenberg gegenüber dem polnischen König als sehr aufschlussreich erwiesen, sowie die Behauptung in einer i. J. 1453 abgefassten Denkschrift des Ordens, Elbing habe damals dem Orden nicht nur die Rückgabe der Burg verwehrt, sondern auch Ordensangehörige festgesetzt ${ }^{62}$, passt zu dem politischen Verhalten der Neustadt, nicht aber zu dem der Altstadt.

\section{ZUSAMMENFASSUNG}

Die wesentlichen Ergebnisse der vorgestellten Beobachtungen lassen sich wie folgt kurz zusammenfassen: Von den zwischen 1380 und 1410 im Bürgermeisteramt der Altstadt Elbing nachgewiesenen Personen können über die Hälfte als Vertraute des Ordens gelten. Zudem lassen sich beinahe alle übrigen als ordensnah qualifizieren. Das Verhalten auch der einfachen Ratsleute gibt trotz der dürftigeren Quellenlage keinen Anlass, gegenteilige Vermutungen zu äußern. Es erstaunt demnach nicht, wenn vor diesem personellen Hintergrund die Altstadt Elbing sich schwertat, die machtpolitischen Gegebenheiten zu ertragen, die nach der Schlacht bei Tannenberg eingetreten sind. Ungeachtet der polnisch-litauischen Besetzung des Elbinger Schlosses nahm der Rat der Altstadt heimlich Verbindung mit der Ordensleitung auf. Sie mündete nach dem Abzug des polnischen Belagerungsheeres vor Marienburg in die offene Unterstützung des Ordens ein, sei es mittels Beteiligung an der Belagerung der polnisch-litauischen Burgbesatzung oder sei es mittels Gestellung von Aufgeboten zur Rückeroberung oder Sicherung von Landesteilen durch den Orden. Die Elbinger Teilnahme an den Vorverhandlungen des 1. Thorner Friedens zeigt an, dass das Vertrauensverhältnis zwischen der Altstadt und dem Orden das Vorkriegsniveau erreicht haben dürfte. Anderslautende Nachrichten passen dagegen eher zum politischen Verhalten der Neustadt Elbing als zu dem der Altstadt.

60 Akten der Ständetage Preussens (wie Anm. 32), Nr. 93, S. $141 \mathrm{f}$.

61 Dies geht aus einem Schreiben des Hauskomturs an den Hochmeister hervor, XX. HA Hist. StA Königsberg, OBA Nr. 1604. Das Schreiben ist ins Jahr 1410 zu datieren, denn der Hauskomtur erwähnt den bevorstehenden Besuch des Hochmeisters in der Stadt. Aus den Elbinger Stadtrechnungen geht hervor, dass die Huldigungsbesuch Plauens noch im Rechnungsjahr 1410 stattgefunden hat, Nowa Ksiega (wie Anm. 9), Tl. 1, Nr. 1043.

62 Pelech (wie Anm. 1), S. 53. 
